\title{
Research of Pseudo Random Noise Code and New Code Construction
}

\author{
Ruizhu He \\ National Astronomical Observatories, CAS \\ University of Chinese Academy of Sciences \\ Beijing, China \\ heruizhu11@mail.ucas.ac.cn
}

\author{
Junxia Cui \\ National Astronomical Observatories, CAS \\ Beijing, China \\ cuijx@nao.cas.cn
}

\begin{abstract}
The pseudo-random code is an important part of the satellite navigation and positioning signals, its performance directly affects the accuracy of positioning system. Especially, in navigation and communication integrated system, the system's anti-jamming, anti-multipath fading, anti-intercepted, anti-multiple access and synchronization are all closely related with the pseudo-random sequence.In this paper, we study the characteristics of $\mathrm{PN}$ codes in navigation and communication integration system, including correlation, balance, run length and linear complexity. Furthermore, it puts forward and constructs a novel imLogCheby sequence successfully. The imLogCheby sequence's uncertainty and randomness has been improved comparatively while maintaining the main advantages of the chaotic sequence with a large number and good confidentiality. We also carry out dynamic simulation in Simulink system to verify the feasibility of the new sequences in the integration system of navigation and communication and their advantage performance in system.
\end{abstract}

Keywords- Satellite navigation system; Integration of navigation and communication; $P N$ Code; Spread spectrum communication; Construction of new PN code

\section{INTRODUCTION}

The distance measurement technology based on pseudorandom code is widely used in the field of satellite navigation, and the four well-known satellite navigation systems (GPS, GLONASS, GALILEO and COMPASS) all use this technology[1]. In satellite navigation and positioning system, the pseudo random noise (PN) code has been applied extensively to measure distance, if the signal modulated by PN code is more random or its spectrum shares more bandwidth, and the signal-to-noise ratio is higher, then the deviation of time difference will be less, and the accuracy of the navigation and positioning system will be improved[2-3]. So the study on PN codes has been one of the hot spots of the navigation and positioning system. Meanwhile, one of the technologies on the integration of navigation and communication is the use of spread spectrum communication technology to transmit navigation information and communication content, and PN code is widely used in spreading spectrum communication. The performance of PN code directly affects the communication capacity of the system, the anti-multipath interference capacity and security performance. So the study of PN code has important significance to improve the positioning accuracy, to improve communication quality, and to increase the capacity of a communication system.

The conventional spread-spectrum communication systems are generally used linear PN code generated by shift registers, for instance, $\mathrm{m}$ sequence and Gold code sequence. The two kinds of sequences have some common defects, such as limited number of available code groups and low complexity. With the breakthroughs have been made to the PN code detection technology of DS-CDMA communication system, confidentiality is threatened [4-8]. Chaos is a kind of pseudo randomness nonlinear process to generate spreading sequence which can be used in spread spectrum communication systems. Because of the high degree of initial sensitivity of chaotic systems, the generated code groups of chaotic could be in very large number, and they also have high linear complexity and excellent confidentiality. The application of the chaotic spreading sequences open up a new perspective for spread spectrum communication, especially after the chaos synchronization problems being solved, its engineering application feasibility have been soared up. Thus, the application of chaotic sequences gets more attention, and become a research focus of spread spectrum communication system. But the chaotic sequences also have their shortcomings, which will lead to unsatisfactory performance in the application of chaotic sequence in navigation and positioning system. In this paper, I construct a new sequence which shows more excellent performances. Finally, I also build a system model for dynamic simulation in Simulink to verify the feasibility of this new sequence in navigation and communication fusion system and their performance superiority.

\section{A NEW PN CODE CONSTRUCTION- IMLOGCHEBY SEQUENCE}

By the study and simulation analysis of the four typical chaotic sequences, their correlation, balance, run length and linear complexity can meet the requirements of the CDMA communication system, in particular, their linear complexity achieve the best. So confidentiality of the communication system using chaotic sequences will be very good. The chaotic sequences show an unmatched advantage that they have a sufficient number of sequences. In this paper, I constructs two kind of new sequences that retain the advantages of the existing chaotic sequences, at the same 
time, their autocorrelation characteristics is as good as $\mathrm{m}$ sequences, and cross correlation characteristic is also good just like Gold sequences.

Among the four typical chaotic sequences, I select improved Logistic sequence and Chebyshev sequence to combination with embedded way, in this article, the improved Logistic mapping is embedded in the mapping of the Chebyshev, so to preserve the balance characteristic is nothing to do with the initial value as Chebyshev mapping. And the uncertainty of the sequence will increase over the compound before. The new mapping is called imLogCheby mapping. Here we get imLogCheby sequence and the mapping function is:

$$
\mathbf{x}_{\mathrm{n}+1}=\cos \left(\omega \arccos \left(1-\mu \mathrm{x}_{\mathrm{n}}^{2}\right)\right)
$$

The mapping system remains chaotic movement. Randomness of the sequence and the balance characteristic get some improvement. Because of the large number of qualifiers, users can define conditions and select excellent properties of the sequences to improve the overall performance of the chaotic spreading sequences.

\section{A. Correlation Characteristic of imLogCheby sequences}

The autocorrelation and cross-correlation characteristics of imLogCheby sequences are shown in Figure 1 and Table 1 .
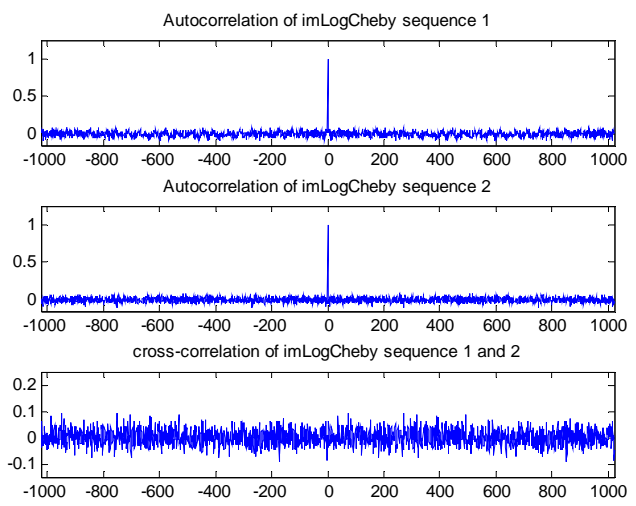

Figure 1. The correlation of the imLogCheby-sequence

TABLE I. THE CORRELATION DATA OF THE IMLOGCHEBY-SEQUENCE

\begin{tabular}{|c|c|c|c|c|c|c|}
\hline Initial value & 0.10 & 0.15 & 0.60 & 0.45 & 0.70 & 0.35 \\
\hline Length & 512 & 512 & 1024 & 1024 & 2048 & 2048 \\
\hline $\begin{array}{c}\text { Max side-lobe of } \\
\text { autocorrelation } \\
\text { value }\end{array}$ & 0.148 & 0.156 & 0.101 & 0.089 & 0.062 & 0.058 \\
\hline $\begin{array}{c}\text { Max cross } \\
\text { correlation value }\end{array}$ & \multicolumn{2}{|c|}{0.148} & \multicolumn{2}{|c|}{0.101} & \multicolumn{2}{|c|}{0.079} \\
\hline
\end{tabular}

From the correlation diagram and data, we see that the autocorrelation function of the new sequence still show a $\delta$ like feature, the spread spectrum signals which have such property enable precision ranging becomes possible, and also help the receiver to capture signals. While the crosscorrelation values of the new sequences are close to zero, quantitatively, when the length of each sequence is close to
1024, the maximum cross-correlation value reaches 0.01 magnitudes optimal, the new sequences' cross correlation and Gold codes' are in an order of magnitude, such a good cross-correlation characteristic enable channels sharing becomes possible.

\section{B. The Balance of imLogCheby sequences}

The relationship of imLogCheby-sequences ' balance and the sequences' length is shown in Figure 2.

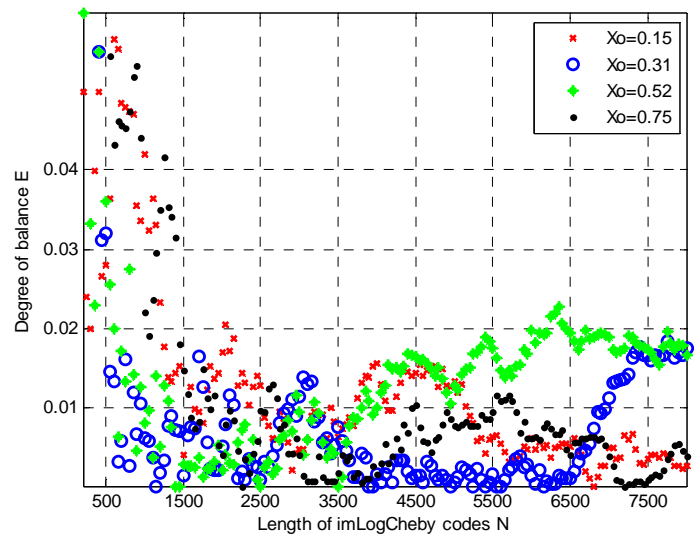

Figure 2. The relationship of imLogCheby-sequences ' balance and the sequences' length

We can see from Figure 2, when sequence's length is greater than 1500, the value of sequence's balance characteristic is less than 0.02 , only a small number of sequences are exception. Due to the large number of new sequences, these exception sequences can be deleted to optimize the overall sequences' performance.

The balance characteristic of the original chaotic sequences is subject to the impact of the initial value, the relationship as shown in Figure 3.
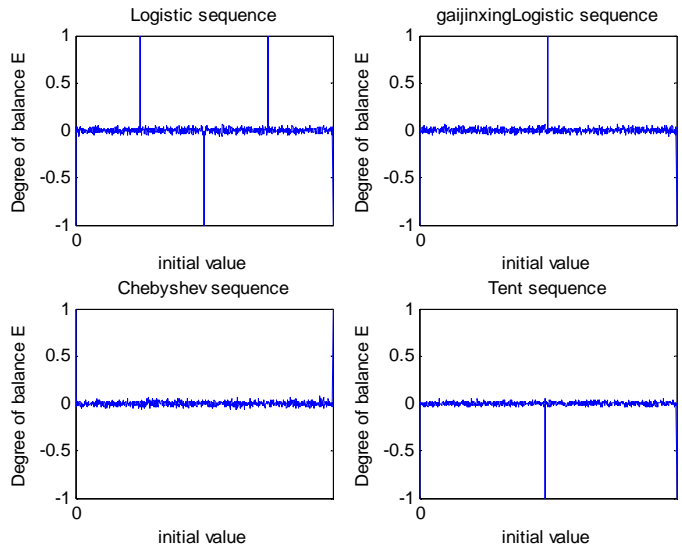

Figure 3. The relationship of chaotic sequences ' initial value and the sequence's balance

In Figure 3, the ranges of the four chaotic sequences are in the $(0,1)$, each sequence's length is 2048 , four mapping fractal parameters were taken 4,2, 64 and 0.49. As can be 
seen, no significant peak point on Chebyshev map, that is to say that the initial value relatively small impact the balancing effects of the Chebyshev sequence; while other three mapping all have some apparent peak points, in these peak points, the balance of sequences will be significantly worse, and should be taken to avoid selecting these initial value in the application. The new sequences I construct in this paper maintain the advantage of the Chebyshev sequence, so the balance of the new sequence is not subject to the influence of the initial value, the relationship as shown in Figure 4.

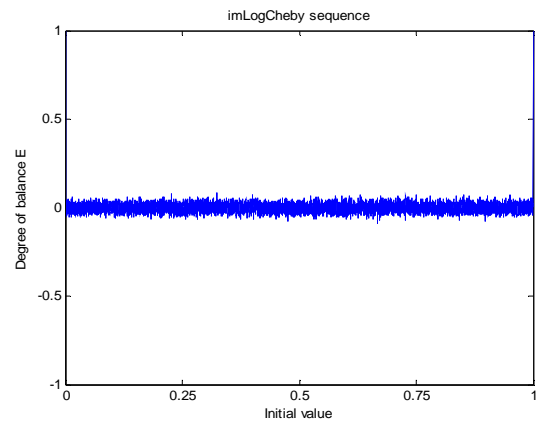

Figure 4. The relationship of imLogCheby-sequence 's initial value and the sequence's balance

\section{The runlength characteristic of imLogCheby sequences}

Beside the correlation and balanced characteristics, sequences' runlength characteristic is also one of important indexes, the runlength characteristic of $\mathrm{m}$-sequence is optimal, and we get the value and compare with the new sequence. The data are shown in Table 2, we can see that imLogCheby sequence runlength characteristic is close to the $\mathrm{m}$-sequence characteristic; they are in line with the law that k-runlength accounting for the total number of $1 / 2^{\mathrm{k}}$.It means that the new sequence's randomness is good.

TABLE II.

TABLE 2. RUN OF SEQUENCES

\begin{tabular}{|c|c|c|c|c|c|}
\hline $\begin{array}{l}\text { The kind } \\
\text { of } \\
\text { sequence } \\
\text { s }\end{array}$ & Length & $\begin{array}{l}\text { Runlength } \\
=1\end{array}$ & $\begin{array}{c}\text { Runlengt } \\
\mathrm{h}=2\end{array}$ & $\begin{array}{c}\text { Runlength }= \\
3\end{array}$ & $\begin{array}{c}\text { Runlengt } \\
\mathrm{h}=4\end{array}$ \\
\hline \multirow{4}{*}{$\begin{array}{c}\mathrm{m}- \\
\text { sequence }\end{array}$} & 255 & 64 & 32 & 16 & 8 \\
\hline & 511 & 128 & 64 & 32 & 16 \\
\hline & 1023 & 256 & 128 & 64 & 32 \\
\hline & 2047 & 512 & 256 & 128 & 64 \\
\hline \multirow{4}{*}{$\begin{array}{l}\operatorname{imLogC} \\
\text { heby } \\
\text { sequence }\end{array}$} & 255 & 64 & 30 & 15 & 9 \\
\hline & 511 & 102 & 61 & 29 & 19 \\
\hline & 1023 & 243 & 125 & 64 & 37 \\
\hline & 2047 & 493 & 253 & 121 & 69 \\
\hline
\end{tabular}

D. The linear complexity of imLogCheby sequences

Finally, study the linear complexity of the new sequence, as shown in Figure 5, the linear complexity of the new sequence is half of the sequence's length. So the new sequence's security and confidentiality are very good.

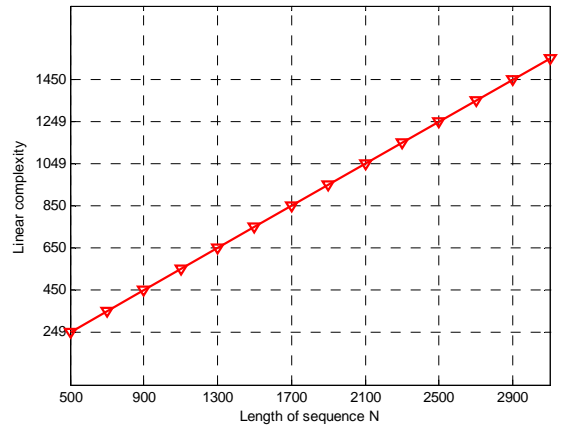

Figure 5. The relationship of imLogCheby-sequence 's length and the linear complexity

The above analysis proved that the new imLogCheby sequences used in navigation and positioning system is feasibility. The new mapping has the characteristic of a greater uncertainty, so obtaining a sufficient number of codes is possible, To concentrated all the sequence has a better performance than the original sequence, the sequences can be preferable to obtain the optimal performance of the set of codes, and to ensure the number of codes in the selected code set are sufficient quantity to meet the requirements of large-capacity CDMA communication system. Such as preferring sequences which have excellent correlation characteristic to improve the ranging accuracy of navigation and positioning system, and to achieve synchronization signal capturing and channel sharing.

\section{System Modeling AND Performance Simulation AND ANALYSIS}

In order to study the system performance of the new pseudo-random sequences, I establish the navigation and communication simulation system used in the DS-CDMA system. The multi-user DSSS communication system used in this paper is shown in Figure 6, the system includes spread-spectrum, modulation, dispreading, correlation operation and judgments and other sectors.

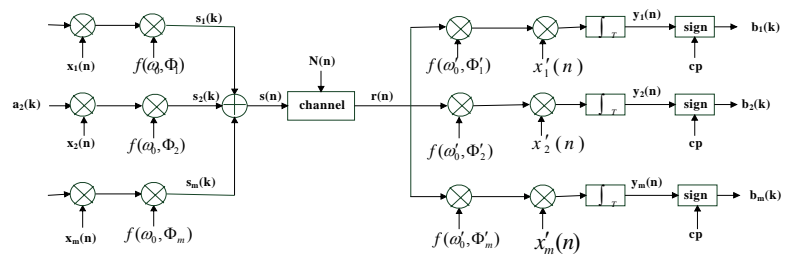

Figure 6. The block diagram of the DS-CDMA communication system

For the study of the anti-multiple access and noise immunity characteristic of pseudo-random sequences in spread spectrum communication system, in the case of ignoring multipath interference, we design of a multi-user spread spectrum communication system based on the correlation receiver and white Gaussian noise channel.

Multi-user spread spectrum communication simulation system shown in figure 7 is constructed based on the correlation receiver and figure 7 . User2 to user6 are package 
subsystems including the user information multiplied by the spreading codes, receiver2 to receiver6, respectively, corresponding to the user's receiver module, the user1 and receiver1 show the un-packaged module.

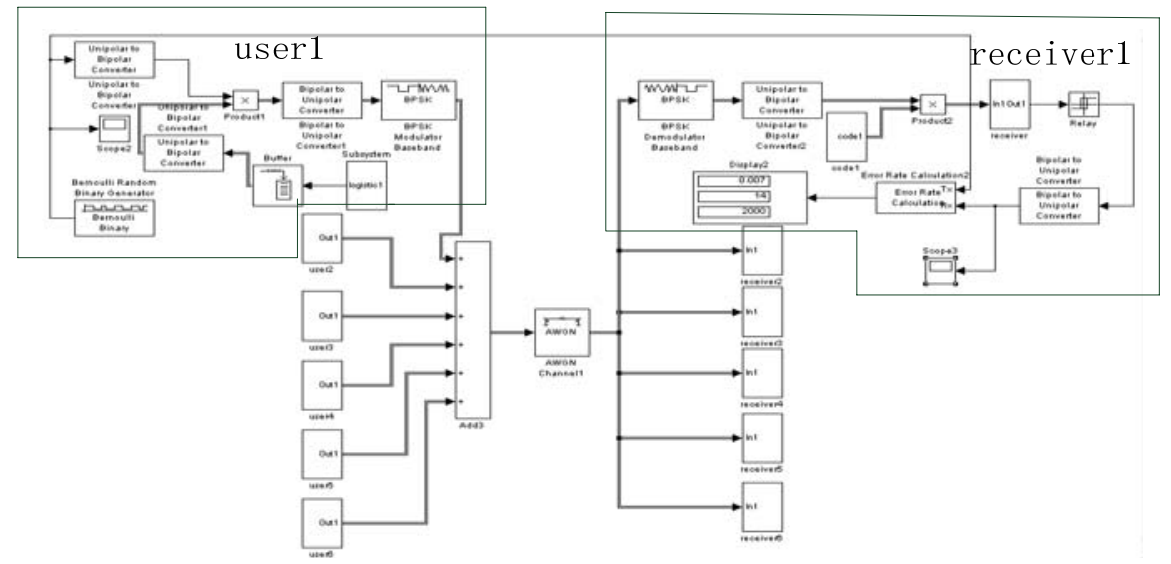

Figure 7. The simulation model of multi-users CDMA communication system

When the SNR is $-10 \mathrm{~dB}$ with imLogCheby sequence as the spreading code, the system simulation result is shown in Figure 8.

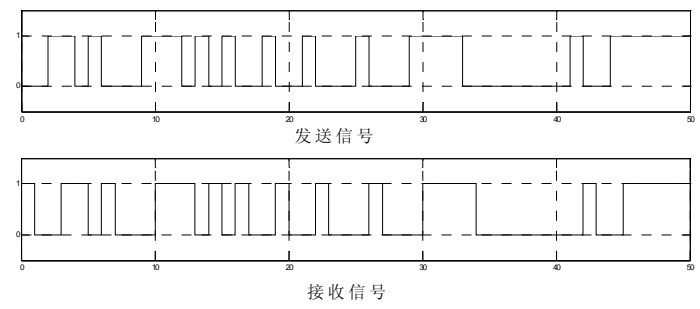

Figure 8. The transmit signals and received signals

Figure 8 shows us the comparison of transmission signal and reception signal, they are nearly identical, the only difference is that the received signal is somewhat delayed (due to the received signal go through the integrator module, somewhat delayed is allowed), that is to say the system used the new sequences is able to receive signals correctly.

\section{CONCLUSION}

In this paper, I put forward a novel chaotic imLogCheby sequence for navigation and communication integrated system, and its characteristics are studied in-depth, including correlation, balance, run length and linear complexity. And the advantages and disadvantages of this new sequence are summarized. Compared with the present pseudo-random sequences, imLogCheby sequence's uncertainty and randomness have been improved to some extent, while maintaining important advantages of chaotic sequences that they have sufficient number and suitable linear complexity. We also simulate and analyze the system performance of new sequence. The result prove the sequences constructed in this paper to have certain advantages, this advantages are not only reflected in the performance of the sequences themselves, but also reflected in the performance of the system.

\section{ACKNOWLEDGMENT}

This work was supported by the National Natural Science Foundation of China (No .61001109)

\section{REFERENCES}

[1] Hu Xiulin, Melting the Ice and Snow equality. GPS and the Galileo signal system design overview of Systems Engineering and Electronics Technology, 2009, 31 (10): 2285-2293.

[2] Tomislav Kos,Mislav Grgic,Lidija Mandic.GPS Modernisation and GNSS Development. 47th International Symposium ELMAR-2005, 2005.8(10):269-273.

[3] Sandhoo K,Turner D, et al. Modernization of the Global Positioning System.In Proceedings of the 13 International Technology Meeting of the Satellite Division of the Institute of Navigation,Salt Lake,U T,Sept.2000 : 2175 2183 .

[4] Hong G K,IICKHO S, Seikho Y,et al.PN Code Acquisition Using Signed-pank-based Nonparametric Detectors in DS/SS Systems. IEEE Transactions on Vehicular Technology. 2001,50(4):172-176.

[5] Wan G B,Kwon H M.PN Code Acquisition Using Smart Antenna for Spread-Spectrum Wireless Communications-part I.Transaction on Vehicular Techology.2003,(1):142-149.

[6] Lu Xiaochun, Chen Qinggang. Satellite navigation and positioning system pseudo-random code. Frequency of time, 2004, 27 (1): 23-31.

[7] Tian Wenting. Spreading code spread spectrum communication system with error correction code. Journal of the National Defense Science and Technology University, 2008.11.

[8] Ma Xinyou. Pseudo-random sequence characteristics of its universal analysis software package. Journal of University of Electronic Science and Technology of China 2001.02. 